Y. C. Minh and E. F. van Dishoeck, eds.

\title{
Diffuse Interstellar Bands
}

\author{
P. J. Sarre and T. R. Kendall \\ School of Chemistry, The University of Nottingham, University Park, \\ Nottingham, NG7 2RD, UK
}

\begin{abstract}
The unidentified diffuse interstellar bands are observed in near-UV, visible and near-IR spectra recorded towards stars which are partially obscured by interstellar dust. Their origin is the longest standing problem in astronomical spectroscopy and dates back to the 1930s when systematic study of the bands first started. Proposals for the carriers range from molecular hydrogen to porphyrins and from colour centres to species adsorbed on grain surfaces. This paper contains a short review of the problem and a discussion of recent possible assignments of some of the bands to transitions of the $\mathrm{H}_{2}, \mathrm{C}_{60}^{+}$and $\mathrm{C}_{7}^{-}$molecules. Observations of ultra-high resolution spectra of diffuse absorption bands, optical diffuse emission bands from the Red Rectangle, and complementary studies of the $3.3 \mu \mathrm{m}$ 'unidentified' infrared (UIR) emission band are described.
\end{abstract}

\section{Introduction}

The search for a solution to the problem of the unidentified diffuse interstellar bands represents a huge challenge in astrochemistry and molecular astrophysics. It is not clear what the solution will bring, but apart from the satisfaction of human curiosity, there is certainly the potential to gain insight into the properties of interstellar material, to provide a new probe of interstellar conditions, and to elucidate the composition and chemistry of cosmic grains.

The diffuse bands appear in spectra recorded towards stars that are reddened by intervening dust which causes the interstellar extinction. The absorptions are described as 'diffuse' because their widths are greater than those arising from transitions in known atoms and small molecules observed along the same lines of sight. There has been controversy since systematic studies of the bands first started in the 1930s (Merrill \& Wilson 1938) as to whether the absorptions arise in free gas phase molecules or in the condensed phase. At present most discussion is focussed on a gas-phase origin though this must remain an open issue until definitive assignments are made. Evidence considered to be in favour of molecules as carriers has grown in recent years and includes the invariance of the wavelengths and narrowness of some of the bands, the lack of polarization across a band, and the discovery that some of the bands are seen in emission from an unusual nebula, the 'Red Rectangle', and an R CrB star V854 Cen at minimum light.

There is generally a good correlation between the band strengths and indices of extinction such as $\mathrm{E}(\mathrm{B}-\mathrm{V})$ as shown, for example, in a recent paper by 
Krelowski et al. (1999) although first recognition of this relation was made many decades ago. There is, however, clear deviation from a strict correlation. A recent study has shown that 44 selected diffuse bands are due to different carriers, although supporting evidence for 'families' of bands (Krelowski \& Walker 1987) was also found (Cami et al. 1997). It certainly appears that the diffuse spectrum arises from a very large number of carriers.

\section{Recent Proposals and Experimental Results}

Proposals for the origin of the bands cover an enormous range, and are described in the comprehensive review by Herbig (1995). One of the most exciting aspects of current research is the focus on possible links between new laboratory and observational data, most notably the attributions of specific bands to transitions of $\mathrm{H}_{2}, \mathrm{C}_{60}^{+}$and $\mathrm{C}_{7}^{-}$.

Sorokin \& Glownia (1996) have assigned a substantial number of bands to transitions between electronically excited states of the hydrogen molecule. Given that $\mathrm{H}_{2}$ is the most abundant molecule in the Universe by far, this appears potentially very attractive. However, major criticisms of this work have been presented by Snow (1998) who has listed eight astrophysical issues to be addressed.

On the general theme of the proposal by Douglas (1977) - that long carbon chains might be carriers-, recent laser experiments have revealed matches, within certain error limits, between vibronic bands of the gas-phase $\mathrm{C}_{7}^{-}$molecule and some of the diffuse bands (Kirkwood et al. 1998; Tulej et al. 1998). The bands discussed include the prominent $\lambda 6269$ absorption which would be the origin band of the A-X system of $\mathrm{C}_{7}^{-}$if confirmed. As part of a wider study of bands at very high resolving power we have recorded the $\lambda 6269$ band towards the star $\mu$ Sgr with a resolving power of $3 \times 10^{5}$ using the Ultra High Resolution Facility (UHRF) at the Anglo Australian Telescope. This is a weakly reddened line of sight with $\mathrm{E}(\mathrm{B}-\mathrm{V})=0.24$ and shows a single narrow $\mathrm{K}$ I profile. The observational data are shown in Figure 1 together with a high-resolution laboratory recording $\left(1 \times 10^{5}\right)$ of the A-X origin band of $\mathrm{C}_{7}^{-}$(Tulej et al. 1998).

The laboratory spectrum was recorded by two-colour laser photodetachment spectroscopy which should give results which are similar, though not necessarily identical to, an absorption spectrum. Although the peak wavelengths are very similar, the spectra of Figure 1 are not identical in peak wavelength, width or shape. The key issue is whether there are reasonable scientific reasons for the discrepancies. Within the astronomical data, it should be recognised that this is only one recording along one line of sight, albeit carefully selected and one which shows a very high degree of fine structure in other bands (Sarre, Miles, \& Scarrott 1995; Kerr et al. 1996,1998). Use of the K I line to provide the radial velocity correction is also generally reliable but not beyond doubt. Within the laboratory data, a key question is the rotational temperature - is it the same as for the diffuse cloud environment? Moreover, it is not clear that the level populations in either laboratory or astronomical spectra can be described by a single temperature. Further work will no doubt reveal the answers to this tantalising situation; observations can be made of other single-cloud lines of sight 


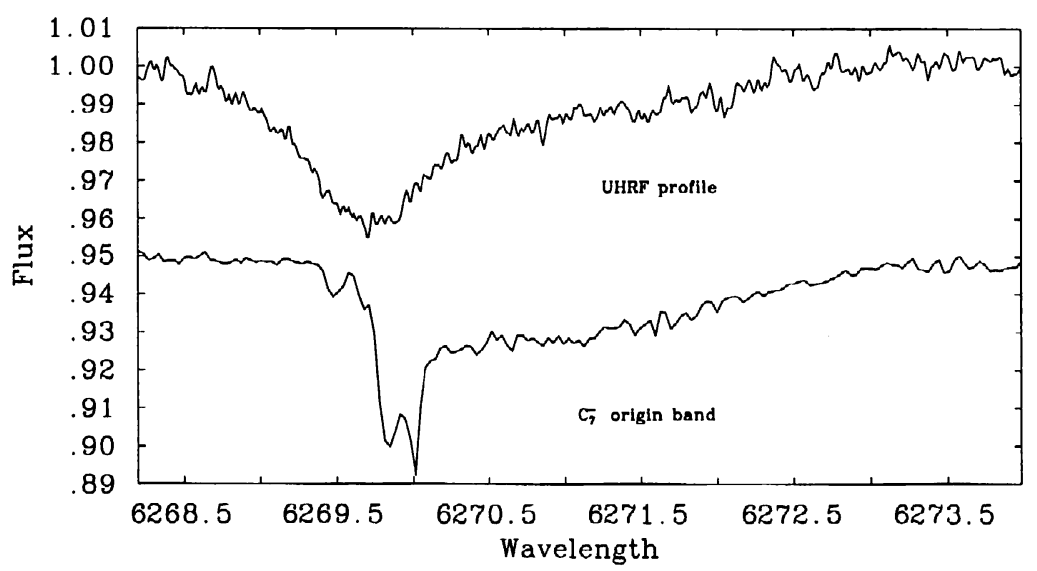

Figure 1. High-resolution spectrum of the $\lambda 6269$ diffuse interstellar band (upper) recorded towards $\mu$ Sgr using the UHRF (RP $3 \times 10^{5}$ ) compared with the laboratory $(\mathrm{A}-\mathrm{X}) 0_{0}^{0}$ band of the $\mathrm{C}_{7}^{-}$molecule (RP $1 \times 10^{5}$ ) recorded by Tulej et al. (1998).

at high resolving power, and in the laboratory a rotationally resolved spectrum is highly desirable, though difficult to achieve.

A further attractive correspondence between laboratory and astrophysical data is the case of $\mathrm{C}_{60}^{+}$(Foing \& Ehrenfreund 1997) which has been studied in the laboratory where it was trapped in a solid neon matrix at low temperature (Fulara, Jakobi, \& Maier 1993). An unsuccessful search for a vibrational satellite band has been made (Jenniskens et al. 1997), but the total available data are not yet sufficiently comprehensive to be clear as to whether the $\mathrm{C}_{60}^{+}$molecule is indeed responsible. The single most important test is the attainment of a 'cold' gas-phase spectrum of the molecule. A recent study in the IR has placed an upper limit on the $\mathrm{C}_{60}^{+}$abundance in some astrophysical environments (Moutou et al. 1999).

\section{Observational Results}

We now consider some of the areas in which we have sought of make a contribution to the diffuse band problem.

\subsection{UHRF spectra}

Observations have been made of many of the stronger and narrower diffuse bands with the Ultra-High Resolution Facility at the Anglo-Australian Telescope (Diego et al. 1995). The first discovery was that of fine structure in the $\lambda 6614$ diffuse band which was found to have a clear triplet structure, and a likely fourth feature on the redward tail (Sarre, Miles, \& Scarrott 1995). Assuming a molecular carrier, rotational contour calculations show that a good match can be obtained for an oblate symmetric top molecule with a rotational constant which 


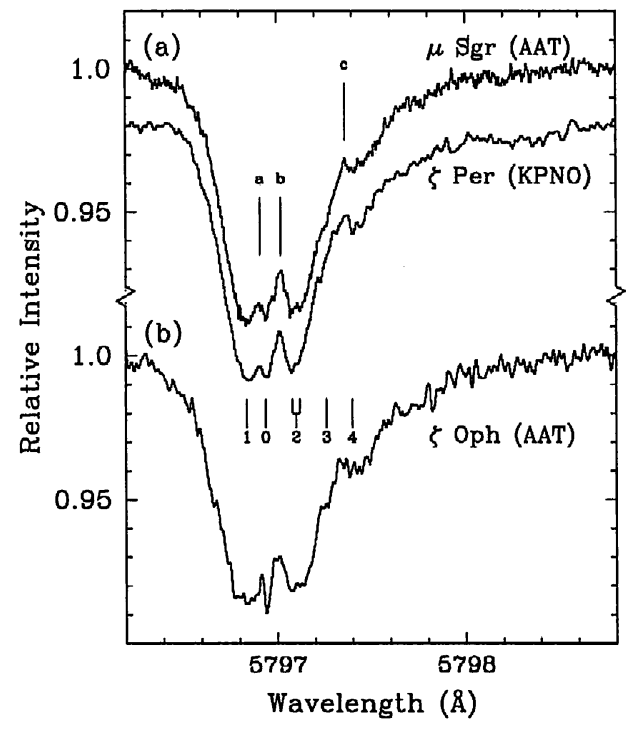

Figure 2. UHRF (AAT) and KPNO coudé feed spectra of the $\lambda 5797$ diffuse interstellar band towards $\mu \mathrm{Sgr}, \zeta$ Per and $\zeta$ Oph. Fine structure features are labelled $0-4$ and a-c (from: Kerr et al. 1998).

corresponds approximately to that of coronene, though an alternative molecule which conforms to this symmetry is a pure carbon ring of between 14 and 30 carbon atoms (Kerr et al. 1996). Data have also been reported by Ehrenfreund \& Foing (1996); from the separations between the attributed $\mathrm{R}$ and $\mathrm{P}$ features it was inferred that these are consistent with PAH molecules of up to 40 carbon atoms, chains of $c a$. 12-18 carbon atoms, 30 carbon atom rings or $\mathrm{C}_{60}$ fullerene compounds. Ultra-high resolution observations of the $\lambda 5797$ band have revealed a hitherto unexpected level of fine structure as shown for $\mu \mathrm{Sgr}, \zeta$ Per and $\zeta$ Oph (see Figure 2). This profile does not so readily lend itself to a description in terms of a triplet $\mathrm{P}, \mathrm{Q}$ and $\mathrm{R}$ structure, although isotopic variations of the same carrier might be able to account for this.

\subsection{Optical spectra of the Red Rectangle}

A particularly interesting astrophysical object is the 'Red Rectangle' which comprises a binary star, surrounded by an oxygen-rich circumbinary disk, and an extended and highly symmetrical biconical-shaped nebula. Excitation of the nebular material results in an extraordinary unidentified optical emission spectrum (Schmidt, Cohen, \& Margon 1980). Measurement of the peak wavelength and width of the major features as a function of offset from the central star along one of the bicone interfaces shows that these properties evolve towards those observed for the corresponding diffuse absorption band observed in diffuse clouds (Sarre, Miles, \& Scarrott 1995). The general structure of the emission has the appearance of molecular vibronic spectrum.

\subsection{IR spectra of the Red Rectangle}

We have also undertaken a study of the spatial distribution of the $3.3 \mu \mathrm{m}$ UIR emission in order to allow comparison with the distribution of the sharp emission (F1) optical feature near $5800 \AA$, the distribution of which was recorded previously by Schmidt \& Witt (1991). In both studies the slit of the spectrograph 


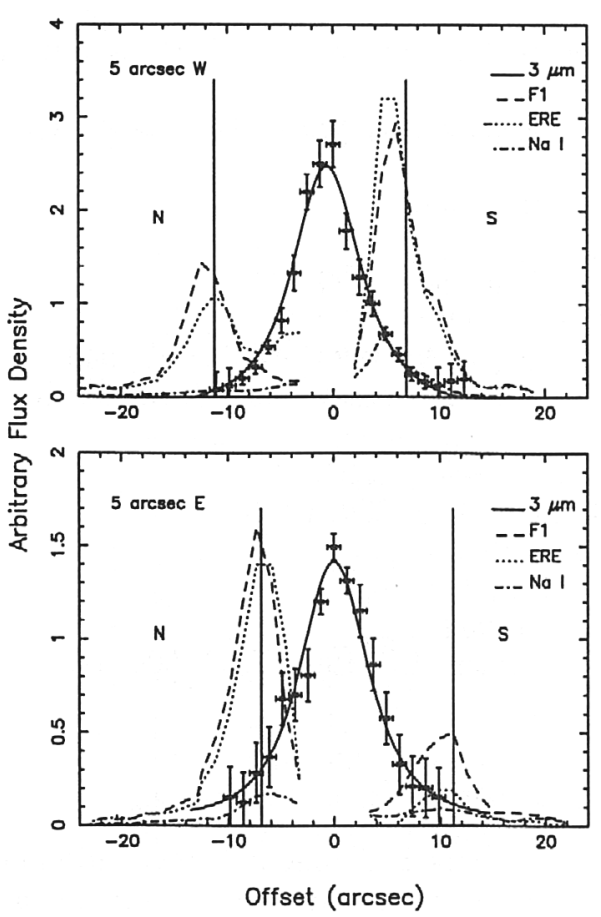

Figure 3. Intensity of the $3.3 \mu \mathrm{m}$ emission measured along the slit $5^{\prime \prime}$ West and $5^{\prime \prime}$ East of HD 44179 compared with that of the unidentified F1 optical feature, Extended Red Emission (ERE) and $\mathrm{Na}$ I as recorded by Schmidt \& Witt (1991). The vertical lines mark the intersections of the slit with the bicone interfaces (from: Kerr et al. 1998).

was oriented parallel to the biconical axis but offset by $5^{\prime \prime}$ East or West. The F1 emission peaks at the two positions where the slit cuts the bicone interface. In sharp contrast the $3.3 \mu \mathrm{m}$ feature has a maximum at the point of closest approach to the star and falls off smoothly with increase in offset along the slit as shown in figure 3 . This is taken to imply that there is at most an indirect relation between the carrier of the $\mathrm{F} 1$ emission and the carrier(s) of the 3.3 $\mu \mathrm{m}$ C-H stretch feature normally attributed to polycyclic aromatic hydrocarbon molecules or material.

Acknowledgments. Support from PPARC, PATT, The Leverhulme Trust, EPSRC and The University of Nottingham is gratefully acknowledged. We thank Professors J P Maier and A N Witt for electronic copies of the high-resolution $\mathrm{C}_{7}^{-}$laboratory spectrum and the Red Rectangle data, respectively.

\section{References}

Cami, J., Sonnentrucker, P., Ehrenfreund, P., \& Foing, B.H. 1997, A\&A, 326, 822

Diego, F., et al. 1995, MNRAS, 272, 323

Douglas, A.E. 1977, Nature, 269, 130

Ehrenfreund, P. \& Foing, B.H. 1996, A\&A, 307, L25

Foing, B.H. \& Ehrenfreund, P. 1997, A\&A, 317, L59

Fulara, J., Jakobi, M., \& Maier, J.P. 1993, Chem. Phys. Lett., 211, 227

Herbig, G.H. 1995, ARA\&A, 33, 19

Jenniskens, P., Mulas, G., Porceddu, I., \& Benvenuti, P. 1997, A\&A, 327, 337 
Kerr, T.H., Hibbins, R.E., Miles, J.R., Fossey, S.J., Somerville, W.B., \& Sarre, P.J. 1996, MNRAS, 283, L105

Kerr, T.H., Hibbins, R.E., Fossey, S.J., Miles, J.R., \& Sarre, P.J. 1998, ApJ, 495,941

Krelowski, J. \& Walker, G.A.H. 1987, ApJ, 312, 860

Krelowski, J., et al. 1999, A\&A, 347, 235

Kirkwood, D.A., et al. 1998, Farad. Disc. Chem. Soc., 109, 109

Merrill, P.W. \& Wilson, O.C. 1938, ApJ, 87, 9

Moutou, C., Sellgren, K., Verstraete, L., \& Léger, A., 1999, A\&A, 347, 949

Sarre, P.J., Miles, J.R., Kerr, T.H., Hibbins, R.E., Fossey, S.J., \& Somerville, W.B. 1995, MNRAS, 277, L41

Sarre, P.J., Miles, J.R., \& Scarrott, S.M. 1995, Science, 269, 674

Schmidt, G.D., Cohen, M., \& Margon, B. 1980, ApJ, 239, L133

Schmidt, G.D. \& Witt, A.N. 1991, ApJ, 383, 698

Snow, T.P. 1998, Farad. Disc. Chem. Soc., 109, 230

Sorokin, P.P. \& Glownia, J.H. 1996, ApJ, 473, 900

Tulej, M., Kirkwood, D. A., Pachkov,M., \& Maier, J.P. 1998, ApJ, 506, L69

\section{Discussion}

E. Herbst: (1) Could you comment on the wavelength change between emission and absorption in the Red Rectangle? (2) Can you compare your astronomical " $\mathrm{C}_{7}^{-}$" observations with those of McCall et al.?

P. J. Sarre: (1) Within the molecular hypothesis, the wavelength shift can be rationalised as arising from a change in the central wavelength of the rotational contour with temperature. However, similar variations in wavelength with temperature are also known for impurities in condensed phase material. (2) I would be interested to compare our observations of the $6269 \AA$ band with their recent results, but I have not yet seen them.

A.N. Witt: Comment: You are to be congratulated on the beautiful result showing the anticorrelated spatial distribution of the sharp feature emission and the $3.3 \mu \mathrm{m}$ emission. The spatial distribution of the ERE in the Red Rectangle follows that of the sharp features very closely, and, therefore, the anticorrelation applies equally to the spatial distribution of ERE and the $3.3 \mu \mathrm{m}$ emission.

D. Williams: Can the complicated profile of the $5797 \AA$ DIB be fitted by rotational modelling?

P. J. Sarre: We have tried to model the $5797 \AA$ profile, but so far with limited success.

T. de Jong: From your modelling of the fine-structure of the DIBs by molecular bands it should be possible to derive theoretical estimates of the abundance of the molecular line carrier(s). What kind of abundances do you find and is this consistent with the carbon budget?

P. J. Sarre: Estimates of the abundances of the carriers have been made, though not by us. Assuming $f \sim 0.1$, the abundance of the carrier of, for example, $\lambda 5780$ $\AA$, is of the order of that for the observed diatomic molecules (Herbig 1993). This is consistent with the carbon budget. 